\title{
Prognostic Value of Triglyceride and Glucose Index for Incident Type 2 Diabetes beyond Metabolic Health and Obesity
}

\author{
Hwi Seung Kim ${ }^{1,2}$, Jiwoo Lee ${ }^{3}$, Yun Kyung Cho ${ }^{4}$, Eun Hee Kim5, Min Jung Lee ${ }^{5}$, Hong-Kyu Kim ${ }^{5}$, Joong-Yeol Park ${ }^{1,2}$, \\ Woo Je Lee ${ }^{1,2}$, Chang Hee Jung ${ }^{1,2}$ \\ ${ }^{1}$ Department of Internal Medicine, Asan Medical Center, University of Ulsan College of Medicine; ${ }^{2}$ Asan Diabetes Center, Asan \\ Medical Center, Seoul; ${ }^{3}$ Department of Internal Medicine, Hallym University Dongtan Sacred Heart Hospital, Hallym \\ University College of Medicine, Hwaseong; ${ }^{4}$ Department of Internal Medicine, Hallym University Sacred Heart Hospital, \\ Hallym University College of Medicine, Anyang; ${ }^{5}$ Department of Health Screening and Promotion Center, Asan Medical \\ Center, University of Ulsan College of Medicine, Seoul, Korea
}

Background: Metabolically healthy obese (MHO) phenotype is metabolically heterogeneous in terms of type 2 diabetes (T2D). Previously, the triglyceride and glucose (TyG) index has been considered for identifying metabolic health and future risk of T2D. This study aimed to evaluate the risk of incident T2D according to obesity status and metabolic health, categorized by four different criteria and the TyG index.

Methods: The study included 39,418 Koreans without T2D at baseline. The risk of T2D was evaluated based on four different definitions of metabolic health and obesity status and according to the baseline TyG index within each metabolic health and obesity group.

Results: During the median follow-up at 38.1 months, 726 individuals developed T2D. Compared with the metabolically healthy non-obese (MHNO) group with low TyG index, the MHO group with high TyG index showed increased risk of T2D in all four definitions of metabolic health with multivariate-adjusted hazard ratios of 2.57 (95\% confidence interval [CI], 1.76 to 3.75 ), 3.72 (95\% CI, 2.15 to 6.43), 4.13 (95\% CI, 2.67 to 6.38), and 3.05 (95\% CI, 2.24 to 4.15), when defined by Adult Treatment Panel III, Wildman, Karelis, and homeostasis model assessment (HOMA) criteria, respectively.

Conclusion: MHO subjects with high TyG index were at an increased risk of developing T2D compared with MHNO subjects, regardless of the definition of metabolic health. TyG index may serve as an additional factor for predicting the individual risk of incident T2D in MHO subjects.

Keywords: Obesity; Metabolic syndrome; Diabetes mellitus, type 2; Obesity, metabolically benign

Received: 9 July 2021, Revised: 9 September 2021, Accepted: 1 October 2021

Corresponding authors: Chang Hee Jung

Department of Internal Medicine, Asan Medical Center, University of Ulsan College of Medicine, 88 Olympic-ro 43-gil, Songpa-gu, Seoul 05505, Korea Tel: +82-2-3010-1418, Fax: +82-2-3010-6962, E-mail: chjung0204@gmail.com

Woo Je Lee

Department of Internal Medicine, Asan Medical Center, University of Ulsan College of Medicine, 88 Olympic-ro 43-gil, Songpa-gu, Seoul 05505, Korea Tel: +82-2-3010-5882, Fax: +82-2-2045-4034, E-mail: 1wjatlas@gmail.com
Copyright $\odot 2021$ Korean Endocrine Society

This is an Open Access article distributed under the terms of the Creative Commons Attribution Non-Commercial License (https://creativecommons.org/ licenses/by-nc/4.0/) which permits unrestricted non-commercial use, distribution, and reproduction in any medium, provided the original work is properly cited. 


\section{INTRODUCTION}

Obesity is a global health burden, as the worldwide prevalence of obesity is constantly increasing [1]. Excess adiposity is infamous for causing cardiometabolic diseases and cancers [1]. However, individuals with a unique obesity phenotype, having a favorable risk profile, referred to as metabolically healthy obese (MHO), have been suggested to have a lower risk of obesity-related diseases [2,3]. Numerous studies have dealt with this select obese population, resulting in conflicting conclusions $[4,5]$.

Regarding the risk of type 2 diabetes (T2D) in MHO individuals, the obesity aspect seems to increase the overall risk compared to metabolically healthy non-obese (MHNO) individuals, while the risk remains considerably low compared to metabolically unhealthy obese (MUO) individuals [5,6]. MHO patients are metabolically heterogeneous in terms of the risk of T2D $[7,8]$. Additionally, the universal definition of $\mathrm{MHO}$ has not yet been established, and different criteria of metabolic health were used in previous studies [9]. The absence of a standard definition of metabolic health may have caused a discrepancy in the results, making it difficult to directly compare the studies and draw a common conclusion [9]. Therefore, there is a significant need to reach a consensus on the best definition of metabolic health, to precisely classify and stratify obese subjects according to risk factors and treat specific groups that would benefit from lifestyle modifications or pharmacological interventions.

Insulin resistance increases the risk of metabolic syndrome, T2D, and cardiovascular diseases [10]. There have been consistent attempts to discover the most efficient indicator of insulin resistance to detect this pathogenic condition in advance $[11,12]$. The product of triglyceride (TG) and fasting plasma glucose (FPG) levels has been suggested as a sensitive marker of insulin resistance [12]. The triglyceride and glucose (TyG) index has proved useful in estimating decreased insulin sensitivity to an extent comparable to the euglycemic-hyperinsulinemic clamp and the homeostasis model assessment of insulin resistance (HOMA-IR) [13,14]. Subsequent studies have suggested that the TyG index could serve as the criterion for distinguishing the metabolic risk subgroups of obesity, especially for identifying the metabolically unhealthy non-obese (MUNO) individuals $[15,16]$. Nevertheless, to our knowledge, no study has yet reported the relationship between the risk of T2D and obesity phenotype, defined by both metabolic health and the TyG index.

In this background, we aimed to evaluate the risk of developing incident T2D according to subgroups of obesity divided by metabolic health status using various definitions in the literature. Moreover, the subgroups of obesity further classified by the TyG index were evaluated in terms of their risk of incident T2D.

\section{METHODS}

\section{Study population}

Study participants were selected from the population that underwent a routine medical examination at the Health Screening and Promotion Center of Asan Medical Center (Seoul, Korea). Between March 2007 and December 2013, 151,759 subjects participated in the examinations, of which 46,480 participated twice. We excluded 3,591 subjects with T2D at baseline and 830 with missing data for serum insulin. Of the remaining 42,059 subjects, 537 with high-sensitivity C-reactive protein (hsCRP) levels greater than $10 \mathrm{mg} / \mathrm{L}$ were excluded to avoid cases of systemic inflammation [7], and 2,104 patients taking lipid-lowering medications were also excluded. Finally, 39,418 subjects (23,087 men and 16,331 women) were included in the analysis. All subjects provided written informed consent, and this study was approved by the Institutional Review Board of Asan Medical Center (IRB No. 2020-1456).

All participants responded to a questionnaire on the medical history of previous diseases and medications and social history, including drinking, smoking, and exercise habits. Drinking habits were categorized as frequency per week (i.e., $<2$ and $\geq 2$ times/week, moderate drinker); smoking habits as past or current, and exercise habits as frequency per week (i.e., $<3$ and $\geq 3$ times/week, physically active) [7].

Incident T2D was diagnosed by documenting either glycated hemoglobin (HbAlc) levels $\geq 6.5 \%$ (48 $\mathrm{mmol} / \mathrm{mol}$ ) or FPG levels $\geq 126 \mathrm{mg} / \mathrm{dL}(7.0 \mathrm{mmol} / \mathrm{L})$ according to the American Diabetes Association criteria [17]. In addition, subjects who reported the use of antidiabetic medications in the questionnaire at the follow-up health examination were also considered to have T2D [7].

\section{Clinical and laboratory measurements}

Details of the clinical and laboratory measurements are available in Supplemental Methods. The HOMA-IR was calculated as the product of fasting serum insulin level $(\mu \mathrm{IU} / \mathrm{mL})$ and FPG (mg/dL), divided by 405 [11]. The TyG index was calculated according to the following formula: $\ln$ (fasting TG $[\mathrm{mg} / \mathrm{dL}] \times$ FPG $[\mathrm{mg} / \mathrm{dL}] / 2)[13,14]$. 


\section{Definitions of metabolic health and obesity status}

Obesity phenotypes were defined by the Asia-Pacific body mass index (BMI) criteria, defined by the World Health Organization Western Pacific Region: non-obese $<25 \mathrm{~kg} / \mathrm{m}^{2}$ and obese $\geq 25$ $\mathrm{kg} / \mathrm{m}^{2}$ [18]. This definition of obesity has been officially chosen by the Korean Centers for Disease Control and Prevention [19].

Four different criteria for metabolic health were implemented in our study (Supplemental Table S1). First, the Adult Treatment Panel III (ATP-III) defined subjects who correspond to less than two of the four criteria as metabolically healthy [20]. The four criteria are as follows: (1) systolic blood pressure (BP) $\geq 130$ $\mathrm{mm} \mathrm{Hg}$ and/or diastolic BP $\geq 85 \mathrm{~mm} \mathrm{Hg}$, or on antihypertensive medication; (2) FPG $\geq 100 \mathrm{mg} / \mathrm{dL}$ (5.6 mmol/L) (impaired fasting glucose [IFG]); (3) $\mathrm{TG} \geq 150 \mathrm{mg} / \mathrm{dL}$ (1.7 mmol/L); (4) high-density lipoprotein cholesterol (HDL-C) $<40 \mathrm{mg} / \mathrm{dL}(1.0$ $\mathrm{mmol} / \mathrm{L})$ in men and $<50 \mathrm{mg} / \mathrm{dL}(1.3 \mathrm{mmol} / \mathrm{L})$ in women [20]. Second, metabolic health defined by the Wildman criteria is having less than two of the six criteria: (1) systolic BP $\geq 130$ $\mathrm{mm} \mathrm{Hg}$ and/or diastolic BP $\geq 85 \mathrm{~mm} \mathrm{Hg}$, or on antihypertensive medication; (2) FPG $\geq 100 \mathrm{mg} / \mathrm{dL}$ (5.6 mmol/L) (IFG); (3) TG $\geq 150 \mathrm{mg} / \mathrm{dL}$ (1.7 mmol/L); (4) HDL-C <50 mg/dL (1.3 mmol/ L); (5) HOMA-IR >90th percentile (i.e., 2.85 in our study); (6) hsCRP >90th percentile (i.e., $2.0 \mathrm{mg} / \mathrm{L}$ in our study) [21]. Third, the Karelis criteria classified people with more than three of the five criteria as metabolically healthy. The five criteria are (1) HOMA-IR $\leq 2.7$; (2) $\mathrm{TG} \leq 150 \mathrm{mg} / \mathrm{dL}$ (1.7 mmol/L); (3) HDL-C $\geq 50 \mathrm{mg} / \mathrm{dL}(1.3 \mathrm{mmol} / \mathrm{L})$; (4) LDL-C $\leq 100 \mathrm{mg} / \mathrm{dL}$ (2.6 mmol/L); and (5) CRP $\leq 3.0 \mathrm{mg} / \mathrm{dL}$ [22]. Last, the HOMA criteria considered individuals within the lower three quartiles of the HOMA index (i.e., 2.02 in our study) as metabolically healthy [2]. The waist circumference (WC) criterion was not applied due to its collinearity with BMI [23]. According to these criteria, the study subjects were sorted into one of four phenotypes: MHNO, MUNO, MHO, and MUO.

\section{Statistical analysis}

Continuous variables are expressed as mean \pm standard deviation. Categorical variables are shown as percentages. The baseline characteristics were compared using one-way analysis of variance or chi-square tests. We performed Cox proportional hazards regression analyses to calculate the hazard ratio (HR) and 95\% confidence interval (CI) for the development of T2D.

First, the risk of incident T2D was evaluated based on the metabolic health and obesity status with the MHNO group as the reference group. Next, the study population was divided into three groups according to the tertile of TyG index (i.e., T1, T2, and T3) for each sex. The lower two tertile groups of each sex were classified as having a low TyG index (i.e., T1 and T2), while the higher tertile group of each sex was classified as having a high TyG index (i.e., T3). The cut-off values for low and high TyG index were 8.89 for males and 8.44 for females. These values were equally applied to all subgroups. The risk for incident T2D was further analyzed according to the baseline TyG index within each metabolic health and obesity group. The MHNO group with low TyG index was used as the reference. In order to assess the prognostic value of TyG index as an additional factor to metabolic health and obesity in predicting incident T2D, Harrell's concordance index (C-index) and net reclassification index (NRI) were calculated using $\mathrm{R}$ statistical software version 3.6.3 (R Foundation for Statistical Computing, Vienna, Austria). All other statistical analyses were performed using SPSS version 18.0 for Windows (SPSS Inc., Chicago, IL, USA). $P<0.05$ was considered statistically significant.

\section{RESULTS}

\section{Baseline characteristics of the study population}

The baseline characteristics of the study participants according to the obesity status and metabolic health based on the ATP-III criteria are shown in Table 1 . Of the 39,418 subjects, $17.5 \%$ were categorized as MHO. Among the 11,949 obese population, $57.7 \%$ were classified as MHO. Compared with MHNO subjects, MHO subjects were more likely to be older, male, current smokers, moderate drinkers, and have a worse metabolic laboratory profile; $\mathrm{MHO}$ individuals were younger and had a more favorable risk profile than MUO individuals. Despite having a higher BMI and WC than MUNO individuals, MHO individuals had lower TG and FPG levels. The baseline characteristics of MHO individuals stratified by TyG index values were evaluated according to the four different definitions of metabolic health (Supplemental Tables S2-S5). In all four criteria for metabolic health, MHO subjects with high TyG index had worse lipid profiles and HOMA-IR than MHO subjects with low TyG index.

The distribution of the TyG index according to the obesity phenotype and metabolic health defined by the ATP-III criteria is demonstrated in Fig. 1A. The TyG index values of the four groups were significantly different. The MUO group had the highest TyG index values, followed by the MUNO, MHO, and MHNO groups. Fig. 1B portrays the prevalence of the TyG index groups among the four phenotypes of metabolic health and obesity status based on the ATP-III criteria. The prevalence of individuals with high TyG index values was 19.7\% (4,511/ 
Table 1. Baseline Characteristics of the Study Participants According to Obesity and Metabolic Health Defined by the Adult Treatment Panel III Criteria

\begin{tabular}{|c|c|c|c|c|c|}
\hline \multirow[b]{2}{*}{ Variable } & \multicolumn{2}{|c|}{ Non-obese } & \multicolumn{2}{|c|}{ Obese } & \multirow[b]{2}{*}{$P$ value } \\
\hline & $\begin{array}{c}\text { MHNO } \\
(n=22,855)\end{array}$ & $\begin{array}{c}\text { MUNO } \\
(n=4,614)\end{array}$ & $\begin{array}{c}\text { MHO } \\
(n=6,895)\end{array}$ & $\begin{array}{c}\text { MUO } \\
(n=5,054)\end{array}$ & \\
\hline Age, yr & $46.7 \pm 8.7$ & $50.9 \pm 8.5$ & $47.8 \pm 8.8$ & $49.0 \pm 8.4$ & $<0.01$ \\
\hline Male sex & $10,072(44.1)$ & $3,521(76.3)$ & $5,041(73.1)$ & $4,453(88.1)$ & $<0.01$ \\
\hline BMI, $\mathrm{kg} / \mathrm{m}^{2}$ & $22.0 \pm 1.9$ & $23.1 \pm 1.5$ & $26.8 \pm 1.7$ & $27.3 \pm 2.0$ & $<0.01$ \\
\hline $\mathrm{WC}, \mathrm{cm}$ & $76.8 \pm 6.8$ & $82.1 \pm 5.8$ & $89.1 \pm 6.0$ & $91.6 \pm 6.0$ & $<0.01$ \\
\hline SBP, $\mathrm{mm} \mathrm{Hg}$ & $112.2 \pm 12.0$ & $126.4 \pm 14.4$ & $118.3 \pm 11.6$ & $127.6 \pm 13.5$ & $<0.01$ \\
\hline DBP, mm Hg & $70.2 \pm 9.0$ & $80.4 \pm 10.1$ & $74.3 \pm 8.7$ & $81.5 \pm 10.0$ & $<0.01$ \\
\hline Current smoker & $4,115(18.0)$ & $1,427(30.9)$ & $2,117(30.7)$ & $1,799(35.6)$ & $<0.01$ \\
\hline Moderate drinker & $1,337(5.8)$ & $572(12.4)$ & $780(11.3)$ & $792(15.7)$ & $<0.01$ \\
\hline Physically active & $5,471(23.9)$ & $929(20.1)$ & $1,401(20.3)$ & 1,091 (21.6) & $<0.01$ \\
\hline Family history of diabetes, $\%$ & $4,218(18.5)$ & $904(19.6)$ & $1,305(18.9)$ & $1,068(21.1)$ & $<0.01$ \\
\hline $\mathrm{HbA} 1 \mathrm{c}, \%$ & $5.3 \pm 0.4$ & $5.4 \pm 0.4$ & $5.4 \pm 0.4$ & $5.5 \pm 0.4$ & $<0.01$ \\
\hline FPG, mg/dL & $91.8 \pm 8.3$ & $102.4 \pm 9.1$ & $94.2 \pm 8.2$ & $102.8 \pm 9.3$ & $<0.01$ \\
\hline $\mathrm{TC}, \mathrm{mg} / \mathrm{dL}$ & $188.4 \pm 31.7$ & $197.2 \pm 33.5$ & $195.9 \pm 32.7$ & $201.2 \pm 34.9$ & $<0.01$ \\
\hline $\mathrm{TG}, \mathrm{mg} / \mathrm{dL}$ & $94.8 \pm 45.7$ & $168.1 \pm 92.3$ & $117.7 \pm 56.0$ & $198.8 \pm 109.3$ & $<0.01$ \\
\hline LDL-C, mg/dL & $116.3 \pm 28.1$ & $125.3 \pm 29.0$ & $127.5 \pm 28.5$ & $129.6 \pm 30.0$ & $<0.01$ \\
\hline HDL-C, mg/dL & $60.9 \pm 13.9$ & $51.8 \pm 13.9$ & $53.6 \pm 11.1$ & $47.2 \pm 11.1$ & $<0.01$ \\
\hline $\mathrm{Cr}, \mathrm{mg} / \mathrm{dL}$ & $0.8 \pm 0.2$ & $0.9 \pm 0.2$ & $0.9 \pm 0.2$ & $1.0 \pm 0.2$ & $<0.01$ \\
\hline Uric acid, mg/dL & $4.8 \pm 1.3$ & $5.7 \pm 1.4$ & $5.7 \pm 1.3$ & $6.3 \pm 1.4$ & $<0.01$ \\
\hline AST, IU/L & $23.1 \pm 16.3$ & $26.0 \pm 13.1$ & $26.2 \pm 13.1$ & $29.2 \pm 17.4$ & $<0.01$ \\
\hline ALT, IU/L & $19.6 \pm 18.1$ & $25.6 \pm 15.9$ & $28.1 \pm 22.2$ & $34.2 \pm 30.0$ & $<0.01$ \\
\hline GGT, IU/L & $20.0 \pm 23.4$ & $34.4 \pm 35.5$ & $30.7 \pm 28.4$ & $44.4 \pm 39.3$ & $<0.01$ \\
\hline Insulin, $\mu \mathrm{IU} / \mathrm{mL}$ & $5.9 \pm 2.9$ & $7.5 \pm 3.6$ & $8.2 \pm 4.3$ & $10.2 \pm 5.3$ & $<0.01$ \\
\hline hsCRP, mg/L & $0.8 \pm 1.1$ & $1.0 \pm 1.2$ & $1.2 \pm 1.3$ & $1.3 \pm 1.4$ & $<0.01$ \\
\hline HOMA-IR & $1.3 \pm 0.7$ & $1.9 \pm 0.9$ & $1.9 \pm 1.1$ & $2.6 \pm 1.4$ & $<0.01$ \\
\hline TyG index & $8.3 \pm 0.4$ & $8.9 \pm 0.5$ & $8.5 \pm 0.4$ & $9.1 \pm 0.5$ & $<0.01$ \\
\hline Incident DM & $157(0.7)$ & $161(3.5)$ & $106(1.6)$ & $301(6.0)$ & $<0.01$ \\
\hline
\end{tabular}

Values are expressed as mean \pm standard deviation or number $(\%)$.

MHNO, metabolically healthy non-obese; MUNO, metabolically unhealthy non-obese; MHO, metabolically healthy obese; MUO, metabolically unhealthy obese; BMI, body mass index; WC, waist circumference; SBP, systolic blood pressure; DBP, diastolic blood pressure; HbA1c, glycated hemoglobin; FPG, fasting plasma glucose; TC, total cholesterol; TG, triglyceride; LDL-C, low-density lipoprotein cholesterol; HDL-C, high-density lipoprotein cholesterol; Cr, creatinine; AST, aspartate aminotransferase; ALT, alanine transaminase; GGT, gamma-glutamyl transferase; hsCRP, high-sensitivity C-reactive protein; HOMA-IR, homeostatic model assessment of insulin resistance; TyG, triglyceride glucose; DM, diabetes mellitus.

$22,855)$ in the MHNO group, $26.2 \%(1,808 / 6,895)$ in the MHO group, $65.3 \%(3,011 / 4,614)$ in the MUNO group, and $75.3 \%$ $(3,806 / 5,054)$ in the MUO group. The distribution and prevalence of the TyG index groups among the four phenotypes of metabolic health and obesity status, as defined by the other three criteria, showed similar patterns as in the ATP-III criteria (Supplemental Figs. S1-S3). The TyG index values were the lowest in the MHNO group, followed by the MHO, MUNO, and MUO groups, and the prevalence of individuals with high TyG index values was higher in metabolically unhealthy groups (MUNO and MUO) than in metabolically healthy groups (MHNO and MHO) (Supplemental Figs. S1-S3).

\section{Risk of incident T2D according to metabolic health and obesity status}

During the median follow-up of 38.1 months (range, 4.8 to 

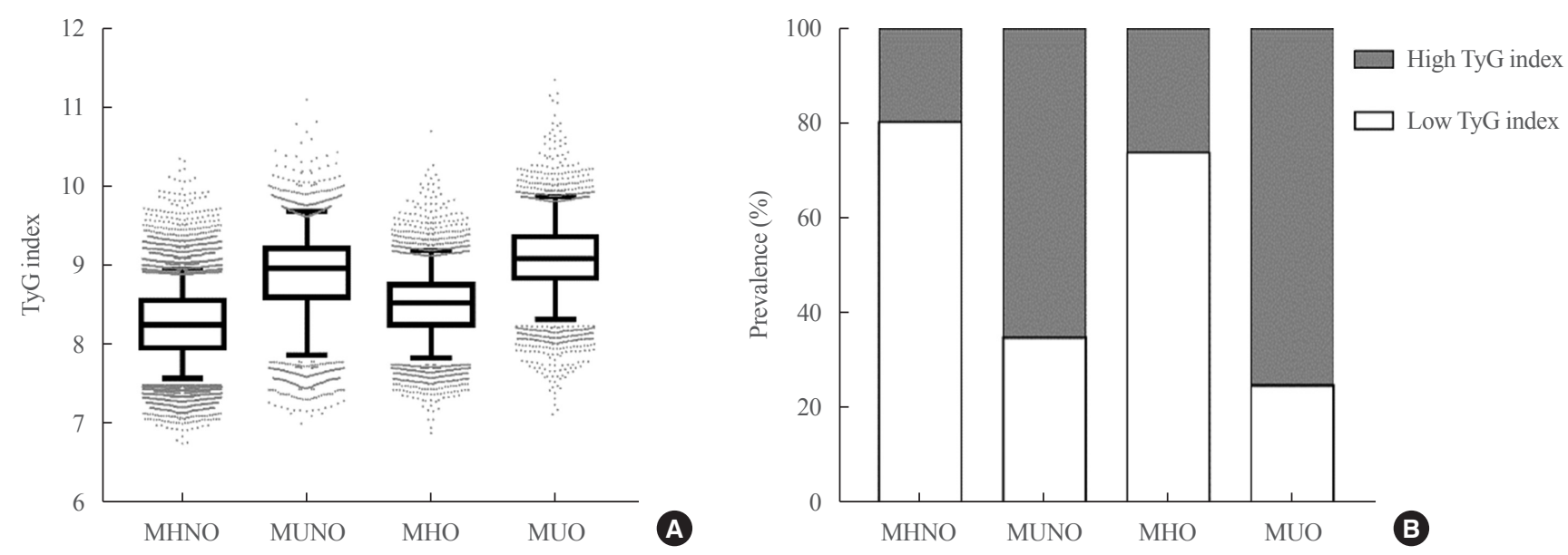

Fig. 1. (A) Distribution of triglyceride glucose (TyG) index, (B) proportion of low and high TyG index groups according to the baseline metabolic health and obesity status defined by the Adult Treatment Panel III (ATP-III) criteria. Error bars display the 5th and 95th percentiles of the TyG index. $P<0.05$ for all comparisons. MHNO, metabolically healthy non-obese; MUNO, metabolically unhealthy non-obese; MHO, metabolically healthy obese; MUO, metabolically unhealthy obese.

Table 2. Prognostic Values of TyG Index as Additional Factor to Metabolic Health and Obesity Status in Predicting Incident Type 2 Diabetes

\begin{tabular}{|c|c|c|c|c|c|c|}
\hline & \multicolumn{2}{|c|}{ Harrell's C-index $(95 \%$ CI $)$} & \multirow{2}{*}{$\begin{array}{l}\text { Difference in } \\
\text { C-indexes }\end{array}$} & \multirow[b]{2}{*}{$P$ value } & \multirow{2}{*}{$\begin{array}{c}\mathrm{NRI}^{\mathrm{a}} \\
(95 \% \mathrm{CI})\end{array}$} & \multirow[b]{2}{*}{$P$ value } \\
\hline & $\begin{array}{l}4 \text { Groups by metabolic health } \\
\text { and obesity status }\end{array}$ & $\begin{array}{c}8 \text { Groups by metabolic health } \\
\text { and obesity status }+ \text { TyG }\end{array}$ & & & & \\
\hline ATP-III & $0.799(0.784-0.814)$ & $0.804(0.789-0.818)$ & 0.004 & $<0.001$ & $0.232(0.131-0.285)$ & $<0.001$ \\
\hline Wildman & $0.794(0.780-0.808)$ & $0.801(0.787-0.815)$ & 0.007 & $<0.001$ & $0.223(0.091-0.309)$ & $<0.001$ \\
\hline Karelis & $0.767(0.751-0.782)$ & $0.785(0.770-0.800)$ & 0.018 & $<0.001$ & $0.248(0.190-0.304)$ & $<0.001$ \\
\hline HOMA & $0.775(0.759-0.790)$ & $0.791(0.776-0.806)$ & 0.016 & $<0.001$ & $0.297(0.258-0.339)$ & $<0.001$ \\
\hline
\end{tabular}

83.8), 726 of 39,418 participants (1.8\%) developed T2D. The incidence rate of T2D based on the ATP-III definition of metabolic health was $0.7 \%(155 / 22,855)$ in the MHNO group, $3.5 \%$ $(161 / 4,614)$ in the MUNO group, $1.6 \%(106 / 6,895)$ in the MHO group, and $6.0 \%$ in the MUO group (301/5,054). The C-index and NRI results of the Cox regression analyses showed that TyG index has an additional prognostic value in predicting the risk of incident T2D beyond metabolic health and obesity status (Table 2) [24,25]. The differences in C-indexes were 0.004, 0.007, 0.018, and 0.016 in ATP-III, Wildman, Karelis, and HOMA criteria, respectively $(P<0.001)$. NRIs in ATP-III, Wildman, Karelis, and HOMA criteria were 0.232 (95\% CI, 0.131 to 0.285), 0.223 ( $95 \%$ CI, 0.091 to 0.309 ), 0.248 (95\% CI, 0.190 to 0.304 ), and 0.297 ( $95 \% \mathrm{CI}, 0.258$ to 0.339 ), respectively.

The HRs for developing incident T2D according to the obesi- ty status and metabolic health defined by the four criteria were evaluated (Table 3, Fig. 2). The adjusted HR of MHO subjects developing incident T2D was 1.39 (95\% CI, 1.05 to 1.83 ), compared with MHNO subjects when defined by the ATP-III criteria (Table 3). The MHO group was associated with significantly higher HRs of developing T2D than the MHNO group, even when the Wildman and HOMA definitions of metabolic health was applied: 1.62 (95\% CI, 1.13 to 2.32), and 1.32 (95\% CI, 1.04 to 1.67) using the Wildman and the HOMA criteria, respectively (Table 3 ). However, the MHO group defined by the Karelis criteria did not show a significant association with an increased risk of incident T2D (HR, 1.34; 95\% CI, 1.00 to 1.81). Moreover, the MUNO group, based on all four criteria of metabolic health, showed overall higher HRs for incident T2D, with the MHNO group as the reference (Fig. 2). The MUO group de- 
Table 3. Hazard Ratios for the Development of Type 2 Diabetes According to Metabolic Health and Obesity Status

\begin{tabular}{|c|c|c|c|c|}
\hline & \multicolumn{4}{|c|}{ Definition of metabolic health } \\
\hline & ATP-III & Wildman & Karelis & HOMA \\
\hline \multicolumn{5}{|l|}{ Incidence } \\
\hline MHNO & $157 / 22,855(0.7)$ & $95 / 19,790(0.5)$ & $141 / 18,637(0.8)$ & $215 / 22,878(0.9)$ \\
\hline MUNO & $161 / 4,614(3.5)$ & $223 / 7,679(2.9)$ & $177 / 8,832(2.0)$ & $103 / 4,591(2.2)$ \\
\hline MHO & $107 / 6,895(1.6)$ & $54 / 4,397(1.2)$ & $83 / 4,127(2.0)$ & $151 / 6,695(2.3)$ \\
\hline MUO & $301 / 5,054(6.0)$ & $354 / 7,552(4.7)$ & $325 / 7,822(4.2)$ & $257 / 5,254(4.9)$ \\
\hline \multicolumn{5}{|c|}{ Unadjusted HRs ( $95 \%$ CI) } \\
\hline MHNO & 1.00 (reference) & 1.00 (reference) & 1.00 (reference) & 1.00 (reference) \\
\hline MUNO & $5.21(4.18-6.49)$ & $6.12(4.82-7.78)$ & $2.63(2.11-3.28)$ & $2.44(1.93-3.08)$ \\
\hline MHO & $2.25(1.76-2.87)$ & $2.53(1.81-3.53)$ & $2.64(2.01-3.46)$ & $2.34(1.90-2.89)$ \\
\hline MUO & $8.96(7.39-10.87)$ & $9.99(7.96-12.52)$ & $5.55(4.55-6.76)$ & $5.50(4.59-6.59)$ \\
\hline \multicolumn{5}{|c|}{ Adjusted HRs $(95 \% \mathrm{CI})^{\mathrm{a}}$} \\
\hline MHNO & 1.00 (reference) & 1.00 (reference) & 1.00 (reference) & 1.00 (reference) \\
\hline MUNO & $3.69(2.91-4.67)$ & $4.08(3.16-5.28)$ & $1.64(1.29-2.07)$ & $1.98(1.56-2.51)$ \\
\hline MHO & $1.39(1.05-1.83)$ & $1.62(1.13-2.32)$ & $1.34(1.00-1.81)$ & $1.32(1.04-1.67)$ \\
\hline MUO & $4.63(3.57-6.00)$ & $5.07(3.79-6.78)$ & $2.26(1.75-2.93)$ & $2.72(2.13-3.46)$ \\
\hline
\end{tabular}

Values are expressed as number (\%).

ATP-III, Adult Treatment Panel III; HOMA, homeostasis model assessment; MHNO, metabolically healthy non-obese; MUNO, metabolically unhealthy non-obese; MHO, metabolically healthy obese; MUO, metabolically unhealthy obese; HR, hazard ratio; CI, confidence interval.

${ }^{a}$ Adjusted for age, sex, waist circumference, systolic blood pressure, drinking, smoking, exercise, family history of diabetes, alanine aminotransferase, gamma-glutamyl transferase, high-sensitivity C-reactive protein, and low-density lipoprotein cholesterol.
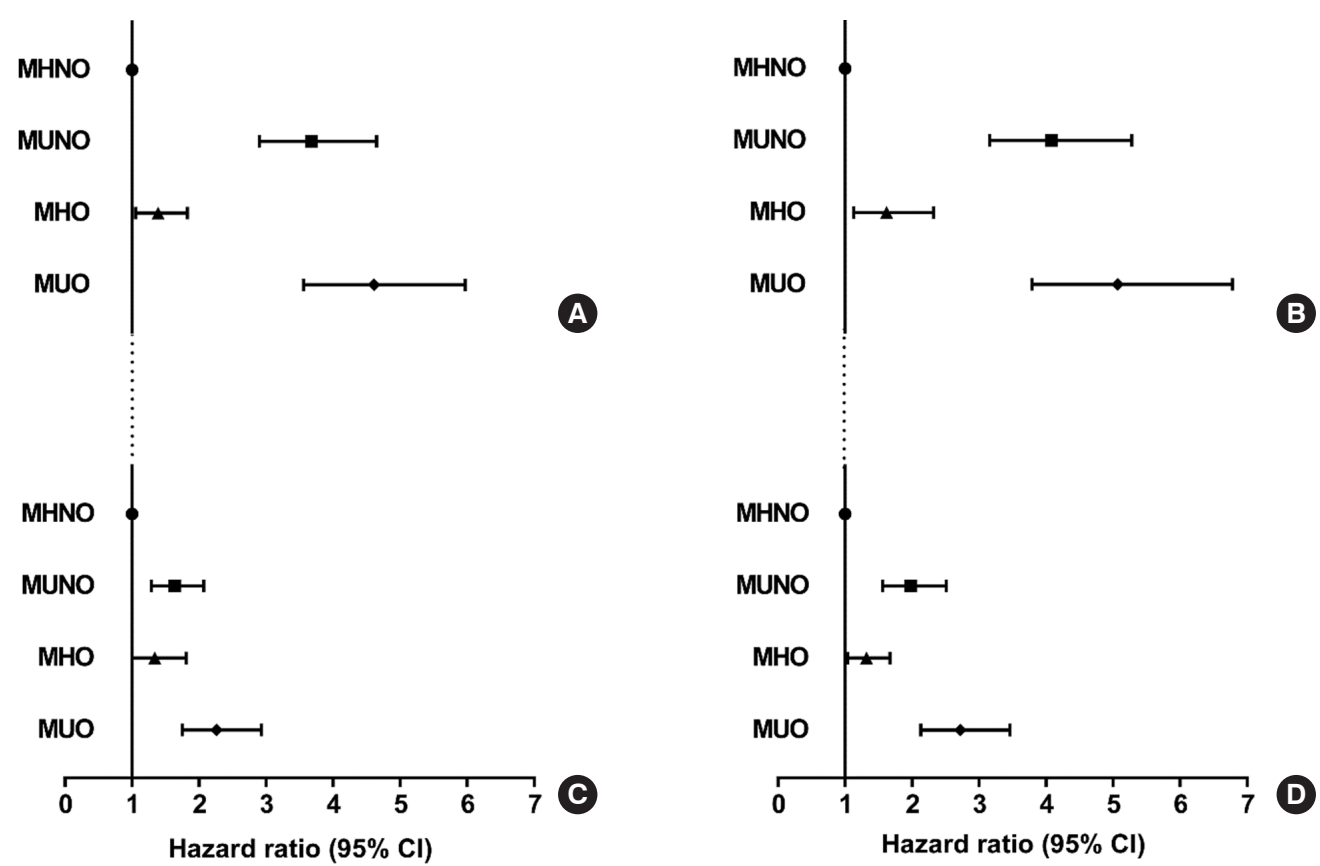

Fig. 2. Hazard ratios for incident type 2 diabetes according to metabolic health and obesity status defined by the four criteria used in the study: (A) Adult Treatment Panel III (ATP-III) criteria, (B) Wildman criteria, (C) Karelis criteria, and (D) homeostasis model assessment (HOMA) criteria. MHNO, metabolically healthy non-obese; MUNO, metabolically unhealthy non-obese; MHO, metabolically healthy obese; MUO, metabolically unhealthy obese; CI, confidence interval. 
fined by all four criteria was associated with the highest value of HR for developing T2D (Table 3, Fig. 2).

\section{Risk of incident T2D according to metabolic health and obesity status classified by TyG index}

The incidence rate of developing T2D was further analyzed by the TyG index in addition to the metabolic health and obesity status (Fig. 3). As demonstrated in Fig. 2, based on the ATP-III criteria, the MUO group with high TyG index had the highest incidence rate $(6.6 \%)$, while the MHNO group with low TyG index had the lowest incidence rate of T2D (0.6\%). The incidence of T2D in the MHO group with high TyG index was higher than that in the MHO group with low TyG index but lower than that of the metabolically unhealthy groups (MUNO and MUO). The Wildman criteria showed a similar pattern as the ATP-III criteria, with the incidence rate of T2D sequentially increasing from MHNO to MUO groups (Fig. 3A, B). However, based on the Karelis and HOMA criteria, the MHO group with high TyG index showed a higher incidence rate of T2D than the MUNO group with high TyG index (Fig. 3C, D).

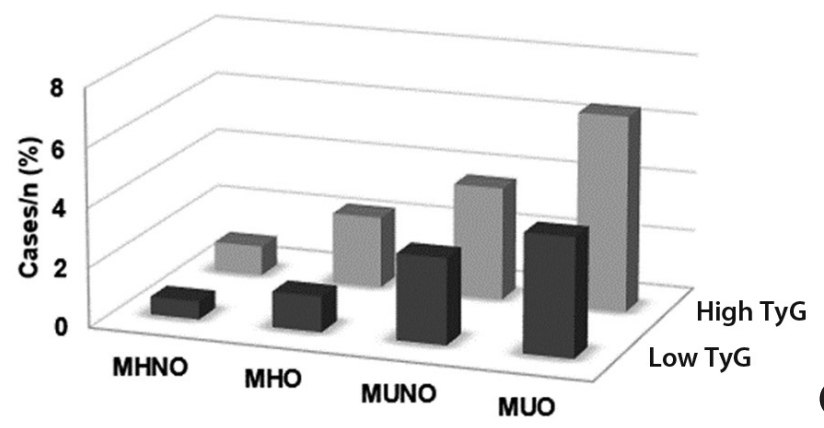

A

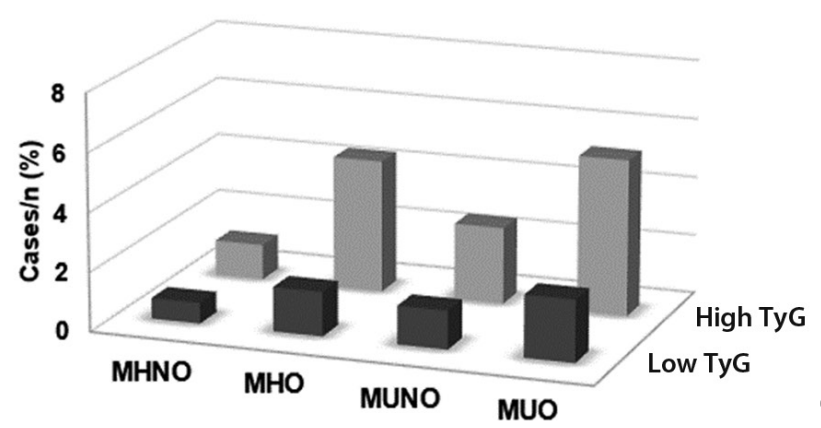

C
The HRs for incident T2D according to the eight groups classified by the metabolic health and obesity phenotype and TyG index were also evaluated (Table 4, Fig. 4). Among the MHO groups defined by all four criteria, the high TyG index subgroups showed significantly higher HRs for incident T2D, with the MHNO group with low TyG index as the reference. The adjusted HRs of the MHO group with high TyG index were 2.57 (95\% CI, 1.76 to 3.75$), 3.72$ (95\% CI, 2.15 to 6.43$), 4.13$ (95\% CI, 2.67 to 6.38 ), and 3.05 (95\% CI, 2.24 to 4.15$)$, using the ATP-III, Wildman, Karelis, and HOMA criteria, respectively (Table 4, Fig. 4). The MUO with high TyG index group was associated with the highest risk of diabetes using the ATP-III, Wildman, and HOMA criteria, while the MHO with high TyG index group showed the highest HR using the Karelis criteria (Table 4, Fig. 4).

Furthermore, the risk of developing T2D in the MHO group classified into low or high TyG index with respect to the MHNO group was assessed (Supplemental Table S6). The adjusted HRs of the MHO group with high TyG index were 2.28 (95\% CI, 1.58 to 3.27$), 3.27$ (95\% CI, 1.91 to 5.57$), 3.39$ (95\% CI, 2.22
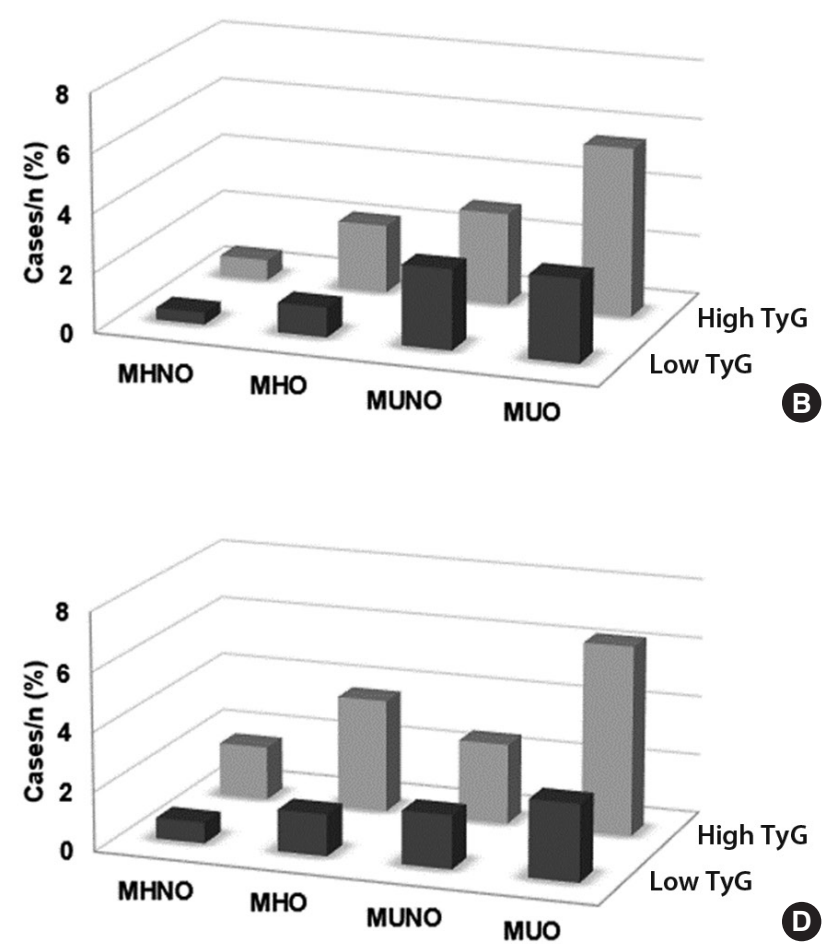

Fig. 3. Incidence rate of type 2 diabetes based on the triglyceride glucose (TyG) index group and metabolic health and obesity phenotype defined by the four criteria used in the study: (A) Adult Treatment Panel III (ATP-III) criteria, (B) Wildman criteria, (C) Karelis criteria, and (D) homeostasis model assessment (HOMA) criteria. MHNO, metabolically healthy non-obese; MHO, metabolically healthy obese; MUNO, metabolically unhealthy non-obese; MUO, metabolically unhealthy obese; 
Table 4. Hazard Ratios for the Development of Type 2 Diabetes According to Metabolic Health and Obesity Status and TyG Index

\begin{tabular}{|c|c|c|c|c|}
\hline & \multicolumn{4}{|c|}{ Definition of metabolic health } \\
\hline & ATP-III & Wildman & Karelis & HOMA \\
\hline \multicolumn{5}{|l|}{ Incidence } \\
\hline \multicolumn{5}{|l|}{ MHNO } \\
\hline Low TyG & $113 / 18,344(0.6)$ & $74 / 16,751(0.4)$ & $111 / 16,130(0.7)$ & $118 / 17,615(0.7)$ \\
\hline High TyG & $44 / 4,511(1.0)$ & $21 / 3,039(0.7)$ & $30 / 2,507(1.2)$ & $97 / 5,263(1.8)$ \\
\hline \multicolumn{5}{|l|}{ MUNO } \\
\hline Low TyG & $47 / 1,603(2.9)$ & $86 / 3,196(2.7)$ & $49 / 3,817(1.3)$ & $42 / 2,332(1.8)$ \\
\hline High TyG & $114 / 3,011(3.8)$ & $137 / 4,483(3.1)$ & $128 / 5,015(2.6)$ & $61 / 2,259(2.7)$ \\
\hline \multicolumn{5}{|l|}{ MHO } \\
\hline Low TyG & $63 / 5,087(1.2)$ & $37 / 3,651(1.0)$ & $54 / 3,489(1.5)$ & $59 / 4,266(1.4)$ \\
\hline High TyG & $44 / 1,808(2.4)$ & $17 / 746(2.3)$ & $29 / 638(4.5)$ & $92 / 2,429(3.8)$ \\
\hline \multicolumn{5}{|l|}{ MUO } \\
\hline Low TyG & $50 / 1,248(4.0)$ & $76 / 2,684(2.8)$ & $59 / 2,846(2.1)$ & $54 / 2,069(2.6)$ \\
\hline High TyG & $251 / 3,806(6.6)$ & $278 / 4,868(5.7)$ & $266 / 4,976(5.3)$ & $203 / 3,185(6.4)$ \\
\hline \multicolumn{5}{|c|}{ Unadjusted HR (95\% CI) } \\
\hline \multicolumn{5}{|l|}{ MHNO } \\
\hline Low TyG & 1.00 (reference) & 1.00 (reference) & 1.00 (reference) & 1.00 (reference) \\
\hline High TyG & $1.56(1.10-2.20)$ & $1.54(0.95-2.50)$ & $1.73(1.16-2.59)$ & $2.71(2.07-3.54)$ \\
\hline \multicolumn{5}{|l|}{ MUNO } \\
\hline Low TyG & $4.97(3.54-6.98)$ & $6.24(4.57-8.51)$ & $1.86(1.33-2.60)$ & $2.74(1.93-3.90)$ \\
\hline High TyG & $6.21(4.79-8.05)$ & $6.92(5.21-9.18)$ & $3.66(2.84-4.72)$ & $4.09(3.00-5.57)$ \\
\hline \multicolumn{5}{|l|}{ МHO } \\
\hline Low TyG & $1.98(1.46-2.69)$ & $2.26(1.52-3.35)$ & $2.21(1.60-3.07)$ & $2.00(1.47-2.74)$ \\
\hline High TyG & $3.99(2.82-5.65)$ & $5.19(3.06-8.78)$ & $6.86(4.56-10.33)$ & $5.52(4.20-7.25)$ \\
\hline \multicolumn{5}{|l|}{ MUO } \\
\hline Low TyG & $6.61(4.74-9.22)$ & $6.42(4.66-8.85)$ & $2.98(2.17-4.09)$ & $4.02(2.91-5.54)$ \\
\hline High TyG & $11.08(8.87-13.84)$ & $13.32(10.31-17.21)$ & $7.93(6.35-9.89)$ & $10.15(8.09-12.74)$ \\
\hline
\end{tabular}

Adjusted HR $(95 \% \mathrm{CI})^{\mathrm{a}}$

MHNO

Low TyG

High TyG

MUNO

\section{Low TyG}

High TyG

MHO

$\begin{array}{ll}\text { Low TyG } & 1.19(0.85-1.67) \\ \text { High TyG } & 2.57(1.76-3.75) \\ \text { MUO } & \\ \text { Low TyG } & 3.37(2.31-4.92) \\ \text { High TyG } & 5.64(4.25-7.49)\end{array}$

1.00 (reference)
$1.46(1.02-2.09)$
$3.59(2.52-5.11)$
$4.14(3.14-5.44)$
$1.19(0.85-1.67)$
$2.57(1.76-3.75)$
$3.37(2.31-4.92)$
$5.64(4.25-7.49)$

1.00 (reference)
$1.55(0.95-2.53)$
$3.97(2.86-5.51)$
$4.57(3.40-6.15)$
$1.40(0.93-2.13)$
$3.72(2.15-6.43)$
$3.19(2.21-4.60)$
$6.79(4.96-9.30)$

1.00 (reference)

$1.91(1.26-2.89)$

$1.12(0.79-1.58)$

$2.29(1.75-2.98)$

$1.12(0.79-1.60)$

$4.13(2.67-6.38)$

$1.23(0.86-1.76)$

$3.30(2.49-4.37)$
1.00 (reference)

$2.30(1.75-3.03)$

$2.32(1.62-3.30)$

$3.13(2.28-4.30)$

$1.16(0.83-1.62)$

$3.05(2.24-4.15)$

$2.09(1.45-3.00)$ $5.24(3.92-6.99)$

Values are expressed as number (\%).

TyG, triglyceride glucose; ATP-III, Adult Treatment Panel III; HOMA, homeostasis model assessment; MHNO, metabolically healthy non-obese; MUNO, metabolically unhealthy non-obese; MHO, metabolically healthy obese; MUO, metabolically unhealthy obese; HR, hazard ratio; CI, confidence interval.

${ }^{a}$ Adjusted for age, sex, waist circumference, systolic blood pressure, drinking, smoking, exercise, family history of diabetes, alanine aminotransferase, gammaglutamyl transferase, high-sensitivity C-reactive protein, and low-density lipoprotein cholesterol. 

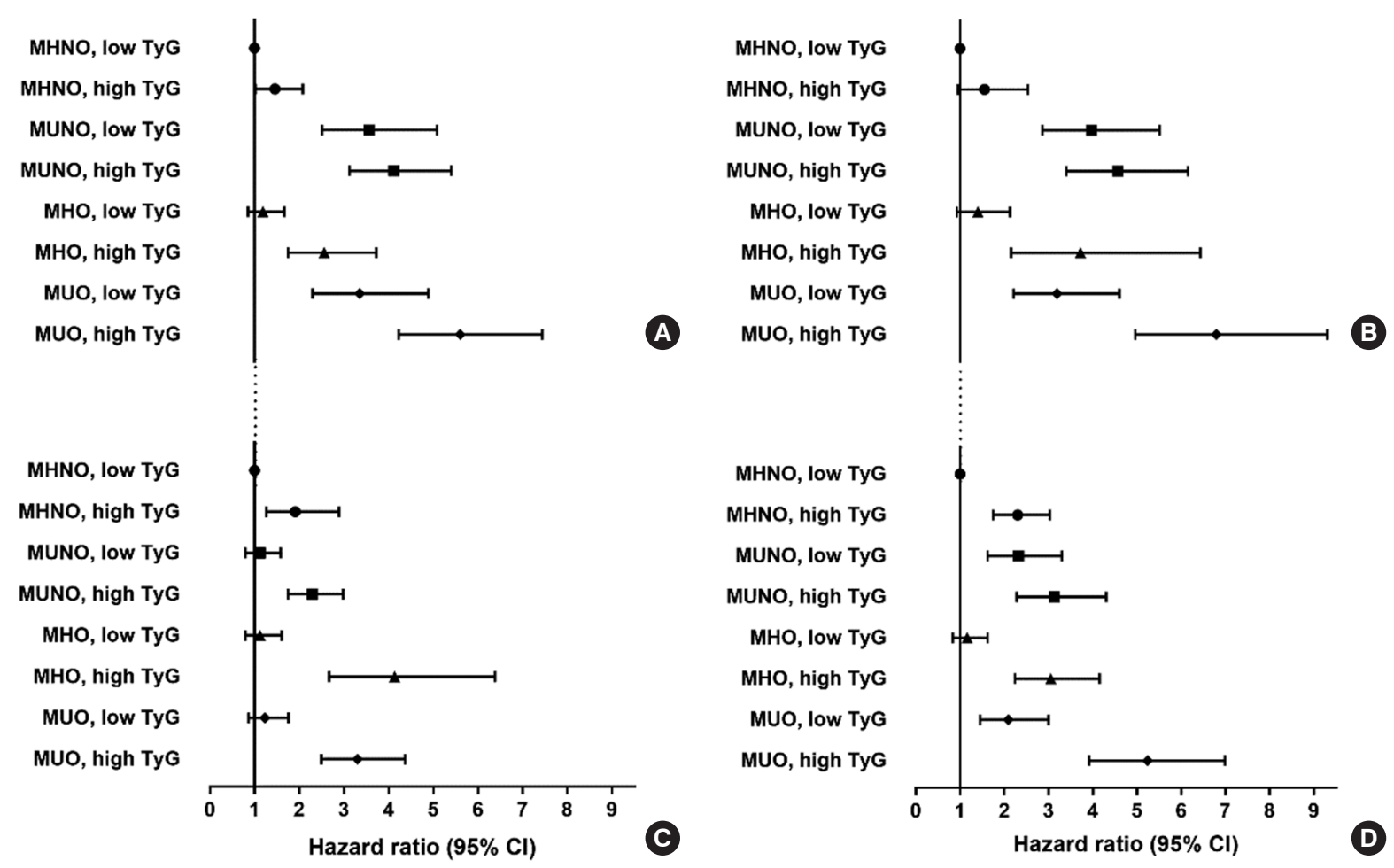

Fig. 4. Hazard ratios for incident type 2 diabetes according to the triglyceride glucose (TyG) index group and metabolic health and obesity status defined by the four criteria used in the study: (A) Adult Treatment Panel III (ATP-III) criteria, (B) Wildman criteria, (C) Karelis criteria, and (D) homeostasis model assessment (HOMA) criteria. MHNO, metabolically healthy non-obese; MUNO, metabolically unhealthy non-obese; MHO, metabolically healthy obese; MUO, metabolically unhealthy obese; CI, confidence interval.

5.17), and 2.12 (95\% CI, 1.61 to 2.79), applying the ATP-III, Wildman, Karelis, and HOMA criteria, respectively. However, the MHO group with low TyG index was not significantly associated with an increased risk of incident T2D in all four criteria (Supplemental Table S6).

\section{DISCUSSION}

In this large population-based study, we analyzed the risk of T2D according to the status of metabolic health and obesity, using four different metabolic health criteria and the TyG index. $\mathrm{C}$-index differences were statistically significant, and NRIs were significantly increased, thereby presenting the TyG index as a valuable additional factor for predicting the risk of T2D. Compared with the MHNO phenotype, the MHO phenotype was associated with an increased risk of incident T2D, irrespective of the definition of metabolic health. Our results indicate that MHO status is not a "benign condition." When the TyG index was considered together with the metabolic health and obesity status, the MHO group with high TyG index showed an increased risk of T2D according to all four definitions of metabol- ic health, whereas the HR of developing incident T2D in the MHO group with low TyG index was insignificant (Table 4, Fig. 4). These findings provide evidence for the heterogeneity of the $\mathrm{MHO}$ group regarding incident $\mathrm{T} 2 \mathrm{D}$ risk and suggest that the TyG index could be an additional useful predictive factor along with metabolic health and obesity status in estimating the risk of incident T2D.

Numerous studies dealt with the relationship between $\mathrm{MHO}$ phenotype and incident T2D, but the results have been conflicting. Previous studies claimed that MHO individuals have a relatively lower risk of incident T2D [3,26,27]. However, more recent meta-analyses and large population-based studies suggested that individuals with $\mathrm{MHO}$ phenotype are not immune to T2D and pose a higher risk of developing it than healthy people with normal weight, indicating that healthy obesity is not a harmless condition, and $\mathrm{MHO}$ is an intermediate state between MHNO and MUO states [28-30].

There are various definitions of metabolic health and MHO phenotype, yet not a consensus on the standard definition $[31,32]$. The criteria vary in components and cut-off values for each component, making it difficult to directly compare the re- 
sults of previous studies [31,32]. Hinnouho et al. [9] showed that mortality risk increased in $\mathrm{MHO}$ individuals, irrespective of the definition of MHO. Furthermore, Kang et al. [33] reported that the elevated risk of incident hypertension in MHO subjects remains consistent in various definitions of metabolic health. This current study analyzed the risk of incident T2D according to various criteria for MHO. The results were in accordance with previous studies [9,33]; the risk of developing T2D was increased in MHO individuals, irrespective of the definition of metabolic health.

Which of the metabolic health and obesity phenotypes contribute more to unfavorable prognosis is debatable. Both MUNO and MHO groups seem to have an increased risk of cardiometabolic diseases and mortality than the MHNO group [34,35]. However, risk comparison between the MUNO and MHO groups has been controversial. A meta-analysis of eight studies showed that the long-term ( $>10$ years) risk of all-cause mortality and cardiovascular events was higher in the MUNO subjects (relative risk [RR], 3.14; 95\% CI, 2.36 to 3.93) than MHO subjects (RR, 1.24; 95\% CI, 1.02 to 1.55 ) [30]. Seven of the eight studies applied the ATP-III criteria to define metabolic health. However, another study that analyzed the risk of hypertension according to different definitions of metabolic health showed inconsistent results [33]. The risk of incident hypertension was higher in MUNO than in MHO using the ATP-III and Wildman criteria, whereas the risk was lower in MUNO than in MHO using the Karelis and HOMA criteria [33]. Our study results were analogous to the meta-analysis, as the HRs of incident T2D were overall higher in the MUNO groups than the MHO groups (Table 3). The ATP-III and Wildman criteria include BP as one of the components of metabolic health, while the Karelis and HOMA criteria do not. Moreover, the three criteria besides ATP-III include HOMA-IR in their definitions. Such differences among the four criteria of metabolic health may be responsible for the conflicting results of the studies. This further highlights the need for a shared consensus on the definition of metabolic health.

The idea that healthy obesity is associated with adverse outcomes suggests the possibility that other factors could have impacted the increased risk of incident T2D. Previous studies have suggested that MHO individuals with a high level of systemic inflammation (measured by hsCRP levels) or increased hepatic steatosis (evaluated by fatty liver index) were associated with an elevated risk of incident T2D [7,8]. TyG index was suggested as a criterion for metabolic health and a predicting factor for T2D [16,36]. Lee et al. [16] classified the subjects with normal weight into metabolically healthy and metabolically unhealthy based on the TyG index. The metabolically unhealthy individuals with normal weight were twice as more likely to develop T2D as metabolically healthy, normal weight individuals were [16]. Furthermore, when obese individuals were included, the risk of incident T2D showed a gradual elevation with HRs of 2.26 (95\% CI, 1.25 to 4.07), 3.04 (95\% CI, 1.69 to 5.47), and 4.04 (95\% CI, 2.14 to 7.63$)$ in MHO, MUNO, and MUO phenotypes respectively, with $\mathrm{MHNO}$ as the reference, using the ATP-III criteria to define metabolic health [36]. However, the HRs varied when the ATP-III criteria for metabolic health were applied: 1.65 (95\% CI, 1.10 to 2.46) in MHO, 3.29 (95\% CI, 2.38 to 4.85 ) in MUNO, and 3.79 (95\% CI, 2.44 to 6.89) in MUO [36]. When we applied the ATP-III criteria to define metabolic health, the HRs were comparable to the previous study (Table 3). We further analyzed the risk of T2D by dividing the four metabolic health and obesity state groups according to the TyG index and showed that in MHO individuals, the TyG index could be a useful discriminator for predicting the risk of developing T2D (Table 4). Finally, the risk of T2D was significantly increased in the MHO with high TyG index group with reference to the MHNO group in all four criteria of metabolic health (Supplemental Table S6).

The mechanism explaining the relatively favorable metabolic outcome of the MHO phenotype is not yet fully understood. MHO individuals seem to have preserved insulin sensitivity and a distinct body fat distribution comprising lower visceral and ectopic fat and higher subcutaneous fat [24,37]. Moreover, the adipose tissue function appears to be preserved, with reduced immune cells infiltration into the adipose tissue [37]. In our study, MHO individuals showed lower values of HOMA-IR than MUO individuals and lower values of TyG index than both MUNO and MUO subjects (Table 1). This is consistent with preserved insulin sensitivity in MHO individuals, as shown in previous studies [7,21].

Our study has some limitations. First, the study population consisted of voluntary participants from the health examination, so it may not represent the general Korean population. Second, the data on lifestyle habits were not collected in a verified quantitative manner. This can explain why the results of our study regarding smoking, alcohol, and physical activity did not show the anticipated difference between the groups when divided by the TyG index. Third, this study was retrospectively analyzed, so the confounding factors might not have been fully considered. Lastly, the 2-hour oral glucose tolerance test was not performed; therefore, some individuals might have had diabetes at 
baseline.

Despite such limitations, to our best knowledge, this is the first attempt to employ various definitions of metabolic health to predict the risk of incident T2D. Since the standard definition of MHO has not yet been established, this large-scale analysis presents further evidence that the obesity aspect of the MHO phenotype is consistently and significantly associated with cardiometabolic health, regardless of the definition of metabolic health.

In conclusion, our study demonstrates that $\mathrm{MHO}$ subjects are at an increased risk of developing T2D compared with $\mathrm{MHNO}$ subjects, regardless of the definition of metabolic health. TyG index may serve as an additional factor for predicting the individual risk of incident $\mathrm{T} 2 \mathrm{D}$.

\section{CONFLICTS OF INTEREST}

No potential conflict of interest relevant to this article was reported.

\section{ACKNOWLEDGMENTS}

This work was supported by the Basic Science Research Program through the National Research Foundation of Korea (NRF), funded by the Ministry of Education (NRF-2020R1A2C1101977: Chang Hee Jung). These funding sources had no roles in the design of the study; the collection, analysis, and interpretation of data; the writing of the article; or the decision to submit the article for publication.

\section{AUTHOR CONTRIBUTIONS}

Conception or design: W.J.L., C.H.J. Acquisition, analysis, or interpretation of data: H.S.K., J.L., Y.K.C., E.H.K., M.J.L., H.K.K., J.Y.P., W.J.L., C.H.J. Drafting the work or revising: H.S.K. Final approval of the manuscript: H.S.K., J.L., Y.K.C., E.H.K., M.J.L., H.K.K., J.Y.P., W.J.L., C.H.J.

\section{ORCID}

Hwi Seung Kim https://orcid.org/0000-0001-5113-9895

Woo Je Lee https://orcid.org/0000-0002-9605-9693

Chang Hee Jung https://orcid.org/0000-0003-4043-2396

\section{REFERENCES}

1. GBD 2015 Obesity Collaborators, Afshin A, Forouzanfar
MH, Reitsma MB, Sur P, Estep K, et al. Health effects of overweight and obesity in 195 countries over 25 years. N Engl J Med 2017;377:13-27.

2. Meigs JB, Wilson PW, Fox CS, Vasan RS, Nathan DM, Sullivan LM, et al. Body mass index, metabolic syndrome, and risk of type 2 diabetes or cardiovascular disease. J Clin Endocrinol Metab 2006;91:2906-12.

3. Primeau V, Coderre L, Karelis AD, Brochu M, Lavoie ME, Messier V, et al. Characterizing the profile of obese patients who are metabolically healthy. Int J Obes (Lond) 2011;35: 971-81.

4. Gao M, Lv J, Yu C, Guo Y, Bian Z, Yang R, et al. Metabolically healthy obesity, transition to unhealthy metabolic status, and vascular disease in Chinese adults: a cohort study. PLoS Med 2020;17:e1003351.

5. Bluher M. Metabolically healthy obesity. Endocr Rev 2020;41:405-20.

6. Magkos F. Metabolically healthy obesity: what's in a name? Am J Clin Nutr 2019;110:533-9.

7. Jung CH, Lee MJ, Kang YM, Jang JE, Leem J, Hwang JY, et al. The risk of incident type 2 diabetes in a Korean metabolically healthy obese population: the role of systemic inflammation. J Clin Endocrinol Metab 2015;100:934-41.

8. Jung CH, Kang YM, Jang JE, Hwang JY, Kim EH, Park JY, et al. Fatty liver index is a risk determinant of incident type 2 diabetes in a metabolically healthy population with obesity. Obesity (Silver Spring) 2016;24:1373-9.

9. Hinnouho GM, Czernichow S, Dugravot A, Batty GD, Kivimaki M, Singh-Manoux A. Metabolically healthy obesity and risk of mortality: does the definition of metabolic health matter? Diabetes Care 2013;36:2294-300.

10. Gast KB, Tjeerdema N, Stijnen T, Smit JW, Dekkers OM. Insulin resistance and risk of incident cardiovascular events in adults without diabetes: meta-analysis. PLoS One 2012;7: e52036.

11. Matthews DR, Hosker JP, Rudenski AS, Naylor BA, Treacher DF, Turner RC. Homeostasis model assessment: insulin resistance and beta-cell function from fasting plasma glucose and insulin concentrations in man. Diabetologia 1985;28:412-9.

12. Du T, Yuan G, Zhang M, Zhou X, Sun X, Yu X. Clinical usefulness of lipid ratios, visceral adiposity indicators, and the triglycerides and glucose index as risk markers of insulin resistance. Cardiovasc Diabetol 2014;13:146.

13. Simental-Mendia LE, Rodriguez-Moran M, GuerreroRomero F. The product of fasting glucose and triglycerides

Copyright (C) 2021 Korean Endocrine Society 
as surrogate for identifying insulin resistance in apparently healthy subjects. Metab Syndr Relat Disord 2008;6:299304.

14. Guerrero-Romero F, Simental-Mendia LE, Gonzalez-Ortiz M, Martinez-Abundis E, Ramos-Zavala MG, HernandezGonzalez SO, et al. The product of triglycerides and glucose, a simple measure of insulin sensitivity. Comparison with the euglycemic-hyperinsulinemic clamp. J Clin Endocrinol Metab 2010;95:3347-51.

15. Lee SH, Han K, Yang HK, Kim MK, Yoon KH, Kwon HS, et al. Identifying subgroups of obesity using the product of triglycerides and glucose: the Korea National Health and Nutrition Examination Survey, 2008-2010. Clin Endocrinol (Oxf) 2015;82:213-20.

16. Lee SH, Han K, Yang HK, Kim HS, Cho JH, Kwon HS, et al. A novel criterion for identifying metabolically obese but normal weight individuals using the product of triglycerides and glucose. Nutr Diabetes 2015;5:e149.

17. American Diabetes Association. 2. Classification and diagnosis of diabetes: standards of medical care in diabetes- 2021 . Diabetes Care 2021;44(Suppl 1):S15-33.

18. WHO Expert Consultation. Appropriate body-mass index for Asian populations and its implications for policy and intervention strategies. Lancet 2004;363:157-63.

19. Oh SW. Obesity and metabolic syndrome in Korea. Diabetes Metab J 2011;35:561-6.

20. Expert Panel on Detection, Evaluation, and Treatment of High Blood Cholesterol in Adults. Executive summary of the third report of the National Cholesterol Education Program (NCEP) Expert Panel on detection, evaluation, and treatment of high blood cholesterol in adults (Adult Treatment Panel III). JAMA 2001;285:2486-97.

21. Wildman RP, Muntner P, Reynolds K, McGinn AP, Rajpathak S, Wylie-Rosett J, et al. The obese without cardiometabolic risk factor clustering and the normal weight with cardiometabolic risk factor clustering: prevalence and correlates of 2 phenotypes among the US population (NHANES 1999-2004). Arch Intern Med 2008;168:1617-24.

22. Karelis AD, Rabasa-Lhoret R. Inclusion of C-reactive protein in the identification of metabolically healthy but obese (MHO) individuals. Diabetes Metab 2008;34:183-4.

23. Despres JP. Excess visceral adipose tissue/ectopic fat the missing link in the obesity paradox? J Am Coll Cardiol 2011;57:1887-9.

24. Pencina MJ, D’Agostino RB Sr, Steyerberg EW. Extensions of net reclassification improvement calculations to measure usefulness of new biomarkers. Stat Med 2011;30:11-21.

25. Uno H, Tian L, Cai T, Kohane IS, Wei LJ. A unified inference procedure for a class of measures to assess improvement in risk prediction systems with survival data. Stat Med 2013;32:2430-42.

26. Stefan N, Kantartzis K, Machann J, Schick F, Thamer C, Rittig K, et al. Identification and characterization of metabolically benign obesity in humans. Arch Intern Med 2008; 168:1609-16.

27. Rhee EJ, Lee MK, Kim JD, Jeon WS, Bae JC, Park SE, et al. Metabolic health is a more important determinant for diabetes development than simple obesity: a 4-year retrospective longitudinal study. PLoS One 2014;9:e98369.

28. Bell JA, Kivimaki M, Hamer M. Metabolically healthy obesity and risk of incident type 2 diabetes: a meta-analysis of prospective cohort studies. Obes Rev 2014;15:504-15.

29. Aung K, Lorenzo C, Hinojosa MA, Haffner SM. Risk of developing diabetes and cardiovascular disease in metabolically unhealthy normal-weight and metabolically healthy obese individuals. J Clin Endocrinol Metab 2014;99:462-8.

30. Kramer CK, Zinman B, Retnakaran R. Are metabolically healthy overweight and obesity benign conditions?: a systematic review and meta-analysis. Ann Intern Med 2013; 159:758-69.

31. Phillips CM. Metabolically healthy obesity: definitions, determinants and clinical implications. Rev Endocr Metab Disord 2013;14:219-27.

32. Rey-Lopez JP, de Rezende LF, Pastor-Valero M, Tess BH. The prevalence of metabolically healthy obesity: a systematic review and critical evaluation of the definitions used. Obes Rev 2014;15:781-90.

33. Kang YM, Jung CH, Jang JE, Hwang JY, Kim EH, Park JY, et al. The association of incident hypertension with metabolic health and obesity status: definition of metabolic health does not matter. Clin Endocrinol (Oxf) 2016;85:207-15.

34. Echouffo-Tcheugui JB, Short MI, Xanthakis V, Field P, Sponholtz TR, Larson MG, et al. Natural history of obesity subphenotypes: dynamic changes over two decades and prognosis in the Framingham Heart Study. J Clin Endocrinol Metab 2019;104:738-52.

35. Kim HS, Lee J, Cho YK, Park JY, Lee WJ, Kim YJ, et al. Differential effect of metabolic health and obesity on incident heart failure: a nationwide population-based cohort study. Front Endocrinol (Lausanne) 2021;12:625083.

36. Navarro-Gonzalez D, Sanchez-Inigo L, Fernandez-Montero A, Pastrana-Delgado J, Martinez JA. TyG index change is 
more determinant for forecasting type 2 diabetes onset than weight gain. Medicine (Baltimore) 2016;95:e3646.

37. Stefan N, Haring HU, Hu FB, Schulze MB. Metabolically healthy obesity: epidemiology, mechanisms, and clinical implications. Lancet Diabetes Endocrinol 2013;1:152-62. 